\title{
Gestational Methylazoxymethanol Exposure Leads to NMDAR Dysfunction in Hippocampus During Early Development and Lasting Deficits in Learning
}

Melissa A Snyder, Alicia E Adelman and Wen-Jun Gao

Neuropsychopharmacology (20 I3) 38, I I 42; doi: I0.I 038/npp.20।3.37

Correction to: Neuropsychopharmacology (2013) 38, 328-340; doi: 10.1038/npp.2012.180; published online 12 September 2012
In this article, the author regret that the NIH grant number used in the Acknowledgment is incorrect. The correct grant number is NIH R01MH085666. 